\title{
Short term effects of temperature on risk of myocardial infarction in England and Wales: time series regression analysis of the Myocardial Ischaemia National Audit Project (MINAP) registry
}

\begin{abstract}
Krishnan Bhaskaran, research degree student, ${ }^{1}$ Shakoor Hajat, senior lecturer, ${ }^{2}$ Andy Haines, professor of public health and primary care and director, ${ }^{3}$ Emily Herrett, research degree student, ${ }^{1}$ Paul Wilkinson, reader in environmental epidemiology, ${ }^{2}$ Liam Smeeth, professor of clinical epidemiology'
\end{abstract}

\section{ABSTRACT}

Objective To examine the short term relation between ambient temperature and risk of myocardial infarction. Design Daily time series regression analysis.

Setting 15 conurbations in England and Wales.

Participants 84010 hospital admissions for myocardial infarction recorded in the Myocardial Ischaemia National Audit Project during 2003-6 (median 57 events a day). Main outcome measures Change in risk of myocardial infarction associated with a $1^{\circ} \mathrm{C}$ difference in temperature, including effects delayed by up to 28 days. Results Smoothed graphs revealed a broadly linear relation between temperature and myocardial infarction, which was well characterised by log-linear models without a temperature threshold: each $1^{\circ} \mathrm{C}$ reduction in daily mean temperature was associated with a $2.0 \%(95 \%$ confidence interval $1.1 \%$ to $2.9 \%$ ) cumulative increase in risk of myocardial infarction over the current and following 28 days, the strongest effects being estimated at intermediate lags of 2-7 and 8-14 days: increase per $1{ }^{\circ} \mathrm{C}$ reduction $0.6 \%$ (95\% confidence interval $0.2 \%$ to $1.1 \%)$ and $0.7 \%$ ( $0.3 \%$ to $1.1 \%)$, respectively. Heat had no detrimental effect. Adults aged 75-84 and those with previous coronary heart disease seemed more vulnerable to the effects of cold than other age groups ( $P$ for interaction 0.001 or less in each case), whereas those taking aspirin were less vulnerable ( $P$ for interaction 0.007).

Conclusions Increases in risk of myocardial infarction at colder ambient temperatures may be one driver of cold related increases in overall mortality, but an increased risk of myocardial infarction at higher temperatures was not detected. The risk of myocardial infarction in vulnerable people might be reduced by the provision of targeted advice or other interventions, triggered by forecasts of lower temperature.

\section{INTRODUCTION}

In the light of global climate change the relations between weather and health are of increasing interest.
In several studies ambient outdoor temperature was shown to affect mortality rates in the short term. A study in 11 US cities found a U-shaped relation between temperature and all cause mortality, with mortality decreasing as temperatures increased from the coldest days up to a certain threshold temperature, above which mortality increased with temperature. ${ }^{1} \mathrm{~A}$ similar pattern has been observed in Europe ${ }^{2}$ and in several lower and middle income countries. ${ }^{3}$ Detrimental effects of both hot and cold days have also been associated with cardiovascular mortality. ${ }^{4-6}$

Less commonly investigated has been the short term effect of ambient temperature on risk of myocardial infarction. A recent systematic review suggested compelling evidence of some temperature effect but was inconclusive on the size or direction of the effect: eight of the 12 studies with data from the winter season found a statistically significant increased risk of myocardial infarction at colder temperatures, whereas seven of 13 studies found a statistically significant detrimental effect of heat, with effect estimates ranging from a $7 \%$ to $40 \%$ increase in rates of myocardial infarction on days with the most extreme temperatures. ${ }^{7}$ Only a few studies have controlled for potentially important confounding variables such as air pollution and circulating influenza levels, which could be associated with both temperature and risk of myocardial infarction. Furthermore, concerns were expressed about the specificity of the outcome in some studies owing to the lack of separate validation, with the potential for inclusion of events not related to myocardial infarction.

We examined the short term effects of temperature in 15 conurbations in England and Wales, controlling for important confounders and making use of a large audit database of myocardial infarction events within which the validity of events could be confirmed against marker data from electrocardiographs and laboratories. 


\section{METHODS}

The Myocardial Ischaemia National Audit Project (MINAP) is a national register of all hospital admissions for myocardial infarction and other acute coronary syndromes, with participation of all hospitals in England and Wales that admit patients with these conditions. The identification of admissions is managed at individual hospital level; guidelines recommend a combination of approaches to identify eligible admissions, including biochemistry records (specifically measurements of troponin), admission notes, and discharge slips. The database includes 123 fields covering basic demographic data, timing of onset of symptoms, changes on electrocardiographs, markers of myocardial necrosis, final diagnosis, thrombolytic or other treatment received, and the geographical coordinates of the super output area (an area with a mean population of 1500) containing the patient's place of residence. Also recorded are pre-existing comorbidities, including hypertension, diabetes, and previous cardiovascular events. We included all events with a discharge diagnosis classified as ST elevation myocardial infarction, non-ST elevation myocardial infarction, or troponin positive acute coronary syndrome, occurring among patients residing within one of 15 conurbations in England and Wales (Greater London, West Midlands, Greater Manchester, West Yorkshire, Tyneside, Liverpool, Nottingham, Sheffield, Bristol, Leicester, Potteries, Cardiff, Southampton, Kingston upon Hull, Norwich) during 2003-6. Conurbation boundaries were predefined to match earlier work. ${ }^{8}$ In a separate sensitivity analysis we reran our final model including only those events that could be validated within the Myocardial Ischaemia National Audit Project database against the presence of recorded raised markers (troponin or creatine kinase) or an electrocardiograph trace showing ST elevation or left bundle branch block.

\section{Meteorological data}

We downloaded data on weather, in particular daily minimum and maximum temperature, temperature at 9 am and $3 \mathrm{pm}$, and dewpoint temperature, from the British Atmospheric Data Centre, listed by weather monitoring station and date of measurement. Daily mean temperature was then generated, approximated as the mean of the daily minimum and maximum temperature. We derived daily relative humidity from the measurements of dewpoint and temperature at 9 am and $3 \mathrm{pm}$. When data from more than one station were available in a conurbation, we combined these to one series using the AIRGENE (air pollution and inflammatory response in myocardial infarction survivors: gene-environment interaction in a high risk group) algorithm. ${ }^{9}$ After combining data in conurbations with multiple stations, eight conurbations had missing data. However, 10 complete temperature series were available at a broader level, based on the following regions: North East, North West, Yorkshire and the Humber, East Midlands, West Midlands,
East, London, South East, South West, and Wales. All available monitoring data are drawn from the area to produce a representative series for the whole region. We used these series as a basis for imputing data for days with missing temperature at the narrower level of conurbation. Specifically, for each conurbation we fitted a simple linear regression model over all days in 2003-6, relating daily conurbation temperature to daily regional temperature; this model was estimated using days with no missing data on conurbation temperature and then used to predict conurbation temperature on days with missing data.

\section{Data on pollution and infectious disease levels}

We downloaded data on daily mean levels of particulate matter with diameters less than $10 \mu \mathrm{m}$ (abbreviated to PM10 and measured as mass in $\mu$ g per $\mathrm{m}^{3}$ of air) and ozone, from the UK air quality data and statistics database. To generate a pollution series for each conurbation for inclusion in our models, we used data from background pollution monitors only, located within one of the 15 conurbations (one to 13 monitors per conurbation). Again we used the AIRGENE algorithm to combine data from multiple stations within a conurbation.

As a measure of level of circulating viral infections, we obtained daily counts of laboratory confirmed cases of influenza A and respiratory syncytial virus for each of 10 UK regions, from the UK Communicable Diseases Surveillance Centre at the Health Protection Agency.

\section{Statistical analysis}

We carried out an ecological time series regression analysis: the daily number of myocardial infarction events was the outcome in a generalised linear model with Poisson error structure, and with scale variables set to the Pearson $\chi^{2}$ statistic divided by the residual degrees of freedom to model overdispersion. ${ }^{1011}$ The main exposure of interest was daily mean temperature. To control for seasonality and long term trend we also included in the model a smooth function of calendar date based on splines, estimated separately for each conurbation. A spline function, defined by piecewise polynomials, has a flexible shape that is useful for modelling unknown and potentially variable seasonal and long term patterns. The smoothness of a spline is a function of the number of degrees of freedom; we chose seven degrees of freedom per calendar year in keeping with previous studies, as a compromise between providing adequate control for unmeasured confounders and leaving sufficient information from which to estimate temperature effects. ${ }^{12}{ }^{13}$ We adjusted the model for day of the week and public holidays, levels of influenza and respiratory syncytial viruses (three categories representing 0,1 , or $\geq 2$ laboratory confirmed cases in the particular conurbation), and PM10 and ozone levels (each modelled at lag days 03 inclusive as there seems to be little evidence of pollution effects at longer lags $\left.{ }^{14}\right)$. We also controlled for 
daily relative humidity (average of the current and previous three days) using a four knot natural cubic spline to allow for non-linearity.

To model the effects of mean temperature we used five lag periods: the average of lag days $0-1,2-7,8-14$, $15-21$, and 22-28. We chose the 0-1 day short lag period because mortality studies suggest that any effects from heat would probably operate with little delay. ${ }^{1516}$ Effects of cold have been reported with longer delays and hence the remaining terms covered delays of up to 28 days, with weekly groupings chosen to allow more precise estimation of effects, and because at longer lags any temperature effects would be unlikely to vary sharply from day to day. To obtain an initial visual estimate of the temperature effect, we included a natural four knot cubic spline for each of the five lagged temperature effects and we used Wald tests to assess the statistical significance of the overall temperature effect (testing all five spline terms) and its non-linearity (testing only the four non-linear terms). We then considered simplified temperature effects with more directly interpretable numerical coefficients - namely, linear and linear threshold temperature models. In the linear threshold temperature models a linear temperature effect only operates below a certain "threshold" temperature, and we fitted the model repeatedly with every possible threshold from the fifth to the 95 th centile of mean daily temperature in $1^{\circ} \mathrm{C}$ steps. We also allowed the thresholds to be specified centiles of temperature within the conurbation, assessing the fifth, 10th, 15th . . 95th centiles. These models were compared and we selected the final temperature effect specification by choosing the model with the lowest Akaike information criterion.

We estimated the cumulative effect of temperature by summing (on the log scale) the regression coefficients of the five individual lagged effects. For a given day, this cumulative effect can be interpreted as the total effect of a difference in daily temperature over the current and following 28 days. ${ }^{17}$ We assessed the heterogeneity of the estimated effects of temperature and potential confounders by including interaction terms and using Wald tests to assess their statistical significance.

Finally, we did an exploratory analysis to assess effect modification by age, sex, previous coronary heart disease, previous hypertension, and current aspirin use. We investigated each potential effect modifier separately: the daily number of events was broken down by the factor under consideration, which was itself included in the model as an interaction with the daily temperature. For the purposes of this exploratory analysis, we included only a single temperature term (average of lag days 0-28) to allow the models to fit, given the small numbers of events in some subgroups. The temperature effect from such a model is comparable to the estimate of the cumulative effect over all lag days, as obtained by summing the five lag terms in our main model.

As well as rerunning our final model including only validated myocardial infarction events, we carried out several other sensitivity analyses, modifying the final model. Firstly, we used minimum and then maximum daily temperature in place of mean daily temperature. Secondly, we varied the number of degrees of freedom per year used to define the spline function of date, used to adjust for season and long term trends. Thirdly, we excluded all but the five conurbations with the highest daily event rates - Greater London, Greater Manchester, West Midlands, Tyneside, and West Yorkshire, all of which had median daily events of at least four). Fourthly, to include information from the $9.5 \%$ of observations with missing pollution levels (PM10 or ozone, or both), we used a multiple imputation procedure with five imputations to handle the missing data. For the imputation we used a multivariate normal model for PM10 and ozone levels containing all variables from the final temperature model. Finally, in case of residual autocorrelation in the final model, we added lagged deviance residuals to the model for each conurbation in which significant early residual autocorrelation was seen (as defined by absolute partial autocorrelations of the deviance residuals exceeding 0.05 at lag days $0-3) .{ }^{18}$ For each sensitivity analysis we estimated the temperature effect at each lag, as well as the cumulative temperature effect.

\section{RESULTS}

Between January 2003 and December 2006, 84010 events (median 57 per day, interquartile range 50-64 per day) were recorded within the 15 conurbations of interest (table 1). Greater London had the largest number of events (26607, median 18, interquartile range 15-21, range 5-36 per day) and Kingston upon Hull the least, with events recorded on only $23.5 \%$ of days (407, median 0 , interquartile range $0-0$, range $0-3$ per day). Overall, $35664 / 84010$ (42\%) of myocardial infarction diagnoses were ST elevation myocardial infarction and $74185(88 \%)$ were confirmed by at least one recorded corroborative electrocardiograph trace (for ST elevation myocardial infarction) or raised troponin or creatine kinase levels, or both.

Myocardial infarction events were recorded at a median age of 70 years (interquartile range $58-79$ years) and $64.5 \%(53819 / 83424)$ of patients whose sex was recorded were men. The daily mean temperature ranged from $-3^{\circ} \mathrm{C}$ to $27^{\circ} \mathrm{C}$ across the 15 conurbations, with individual conurbations having a median value of between $9^{\circ} \mathrm{C}$ and $12^{\circ} \mathrm{C}$ during 2003-6 (table 1).

\section{Modelling temperature effects as flexible curves}

Flexible curves representing the combined effect of temperature across all 15 conurbations, adjusted for potential confounders, were compatible with a broadly linear effect of temperature at short lags (days 0-1 and 2-7), with the risk of myocardial infarction increasing at lower temperatures, although the temperature effect was not significant at days $0-1(\mathrm{P}=0.62$, fig 1$)$. At days 8-14 evidence of a temperature effect was strong $(\mathrm{P}=0.006)$; again an increase in the risk of myocardial infarction was seen at lower temperatures, and 


\begin{tabular}{|c|c|c|c|c|c|c|c|}
\hline Conurbation & $\begin{array}{l}\text { Median (range) of daily } \\
\text { mean temperature }\left({ }^{\circ} \mathrm{C}\right)\end{array}$ & $\begin{array}{c}\text { Total } \\
\text { No of Mls }\end{array}$ & $\begin{array}{l}\text { STEMI } \\
(\%)\end{array}$ & $\begin{array}{l}\text { Median (interquartile } \\
\text { range) Mls per day }\end{array}$ & $\begin{array}{l}\text { Men } \\
(\%)\end{array}$ & $\begin{array}{c}\text { Median (interquartile } \\
\text { range) age* }\end{array}$ & $\begin{array}{l}\text { Evidence supporting } \\
\text { diagnosis } \dagger(\%)\end{array}$ \\
\hline Bristol & $11(-2-26)$ & 2376 & $1007(42)$ & $1(0-2)$ & $1519 / 2354(64.5)$ & $71(60-80)$ & $2224(94)$ \\
\hline Cardiff & $12(0-25)$ & 1471 & $507(34)$ & $1(0-2)$ & $857 / 1464(58.5)$ & $75(63-83)$ & $1446(98)$ \\
\hline Greater London & $12(-1-27)$ & 26607 & $12048(45)$ & $18(15-21)$ & $17863 / 26532(67.3)$ & $69(57-79)$ & $23319(88)$ \\
\hline Greater Manchester & $11(-3-26)$ & 12434 & $5139(41)$ & $8(6-11)$ & $7722 / 12424(62.2)$ & $71(59-80)$ & $10492(84)$ \\
\hline Kingston upon Hull & $11(-2-23)$ & 407 & $369(91)$ & $0(0-0)$ & $278 / 406(68.5)$ & $64(56-73)$ & $400(98)$ \\
\hline Leicester & $10(-2-23)$ & 1768 & $969(55)$ & $1(0-2)$ & $1239 / 1768(70.1)$ & $68(56-77)$ & $1685(95)$ \\
\hline Liverpool & $11(-3-24)$ & 4358 & $1674(38)$ & $3(2-4)$ & $2623 / 4355(60.2)$ & $72(61-80)$ & $3775(87)$ \\
\hline Norwich & $10(-2-25)$ & 874 & $339(39)$ & $0(0-1)$ & $605 / 874(69.2)$ & $70(60-77)$ & $863(99)$ \\
\hline Nottingham & $10(-2-23)$ & 1488 & $907(61)$ & $1(0-2)$ & $974 / 1476(66.0)$ & $68(57-77)$ & $1374(92)$ \\
\hline Potteries & $10(-3-25)$ & 2205 & $854(39)$ & $1(1-2)$ & $1437 / 2205(65.2)$ & $72(60-80)$ & $1713(78)$ \\
\hline Sheffield & $10(-2-24)$ & 4903 & $1443(29)$ & $3(2-5)$ & $2891 / 4878(59.3)$ & $72(61-81)$ & 4536 (93) \\
\hline Southampton & $12(0-25)$ & 1259 & $517(41)$ & $1(0-1)$ & $875 / 1259(69.5)$ & $69(58-78)$ & $1248(99)$ \\
\hline Tyneside & $10(-2-23)$ & 8017 & $2409(30)$ & $5(4-7)$ & $4697 / 8010(58.6)$ & $72(60-81)$ & $6717(84)$ \\
\hline West Midlands & $10(-3-24)$ & 9265 & $4827(52)$ & $6(4-8)$ & $6258 / 9245(67.7)$ & $68(57-77)$ & 8646 (93) \\
\hline West Yorkshire & $9(-2-22)$ & 6578 & $2655(40)$ & $4(3-6)$ & $3981 / 6174(64.5)$ & $69(58-77)$ & $5747(87)$ \\
\hline Overall & $11(-3-27)$ & 84010 & $35664(42)$ & $57(50-64)$ & $53819 / 83424(64.5)$ & $70(58-79)$ & $74185(88)$ \\
\hline
\end{tabular}

MIs=myocardial infarctions; STEMI=ST elevation myocardial infarction.

*Data on age available for $81441 / 84010$ (97\%) of diagnoses, and sex for 83 424/84 010 (99\%) of diagnoses.

†Evidence for STEMI diagnoses was defined as either an electrocardiograph trace indicating ST elevation or left bundle branch block, or raised markers (troponin or creatine kinase); evidence on diagnoses for non-ST elevation myocardial infarction was defined as raised markers (troponin or creatine kinase) only.

although the curve suggested a levelling off of the effect at both the lower and the upper extremes of the temperature range, confidence intervals in these regions were wide, reflecting the limited number of days on which these extremes of temperature occurred. At lag days 15-21 and 22-28 we found little evidence of any continuing temperature effect, although in both cases the estimated curves were broadly in the direction of a detrimental effect of cold.

\section{Selection of linear or threshold temperature models}

Simplified specifications of the temperature effect using linear and cold threshold models were considered within the same modelling framework. The Akaike information criterion was minimised by the most parsimonious "all-linear" model (that is, without threshold, Akaike information criterion 67414.1 ); in the optimal threshold model the cold threshold was at the 90th centile of temperature (Akaike information criterion 67414.9 ). This in any case represents close to a linear temperature effect.

\section{Description of effects in final model}

It was estimated that a $1^{\circ} \mathrm{C}$ reduction in temperature on a given day would cumulatively increase the risk of myocardial infarction by $2.0 \%$ (95\% confidence interval $1.1 \%$ to $2.9 \%$ ) over the current and following 28 days, with the strongest effects being estimated at intermediate lags of 2-7 and 8-14 days (relative risk 1.006 (95\% confidence interval 1.002 to 1.011$)$ and 1.007 (1.003 to 1.011), respectively, table 2).

Estimates from the final model also showed evidence of effects of day of the week and PM10 levels. As expected, the risk of myocardial infarctions being reported on weekdays compared with weekends was estimated to increase: compared with Sunday, the risk of a myocardial infarction being recorded on a Monday was increased by $14 \%{ }^{11-17}$ and on Tuesday to Friday by $4-8 \%$. Increases in PM10 levels were associated with a small increase in risk of myocardial infarction on the same day (relative risk 1.001, 95\% confidence interval 1.000 to 1.002 ), although no effect of ozone level was observed $(\mathrm{P}=0.19)$.

Relative humidity did not seem to be associated with risk of myocardial infarction $(\mathrm{P}=0.29)$, although there was a non-significant increase in risk at both low and high humidity values: relative risk 1.03 (95\% confidence interval 0.99 to 1.06$)$ and 1.06 (0.99 to 1.13$)$ at $55 \%$ and $95 \%$ compared with $75 \%$ ).

Finally no evidence was found of effects of public holiday or of influenza or respiratory syncytial virus in the final model although effect estimates were in the direction expected in each case - that is, a reduced risk of myocardial infarctions being recorded on public holidays and an increased risk on days with several laboratory confirmed cases of influenza or respiratory syncytial virus infection.

\section{Variation across conurbations}

The temperature effect across conurbations was not heterogeneous ( $\mathrm{P}$ for interaction 0.43 ). At lag days 2-7 and $8-14$, at which significant combined cold effects were estimated, effect estimates were in a direction suggesting cold effects for 11 and 12 conurbations, respectively; however, the only conurbation in which these effects were individually statistically significant was Greater London (fig 2). Greater London had a median of 18 events a day compared with fewer than eight in all other conurbations and therefore was much better powered to detect these effects. 

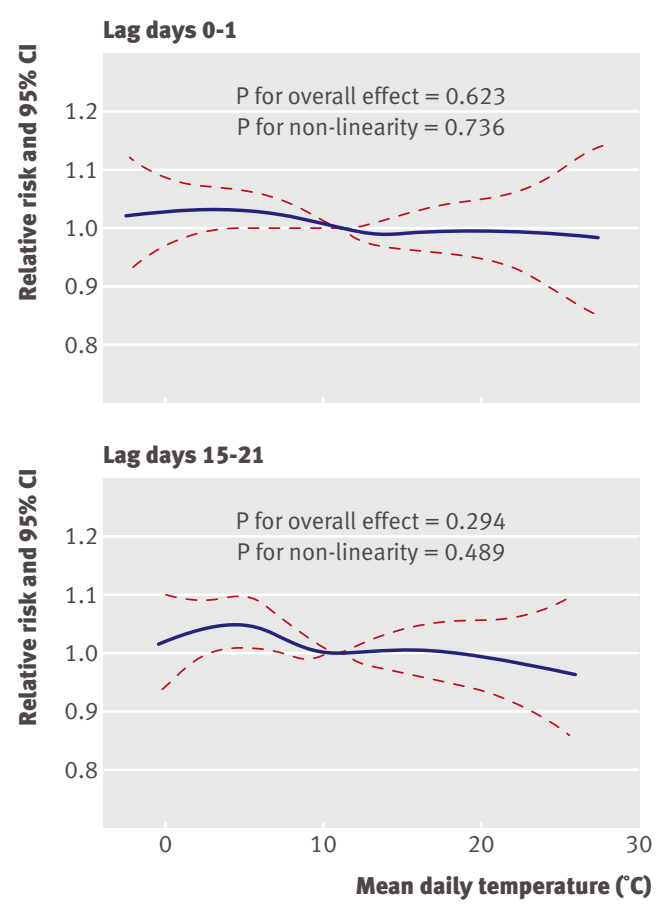
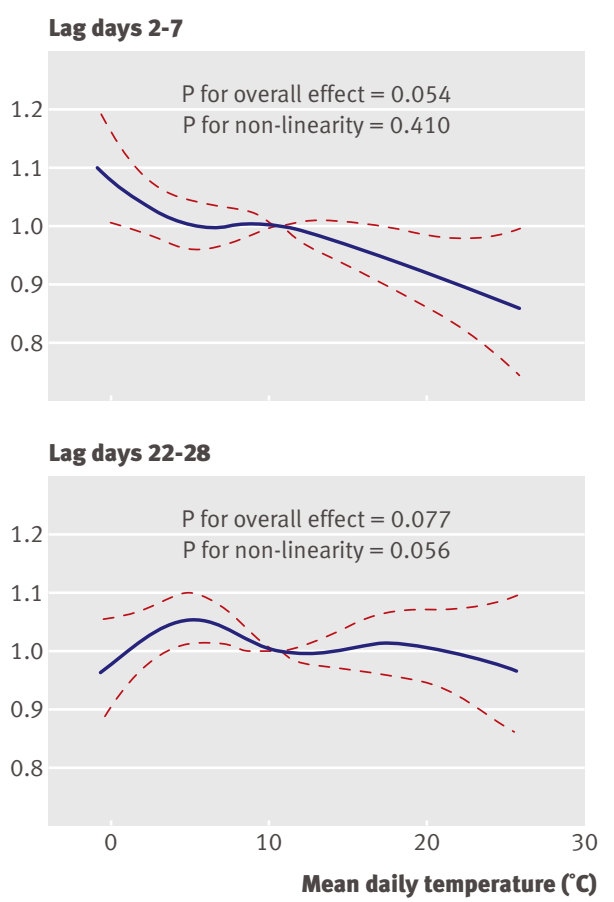

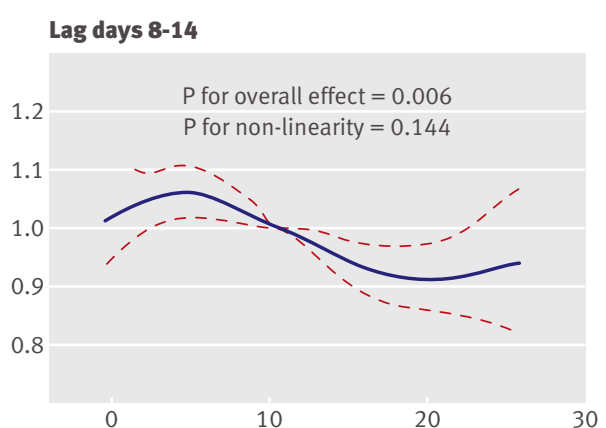

Mean daily temperature $\left({ }^{\circ} \mathrm{C}\right)$

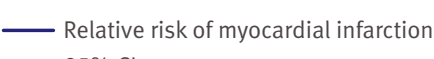
$--95 \% \mathrm{Cl}$

Fig 1| Estimated relative risk of myocardial infarction by temperature in 15 conurbations combined, from a model including all five lag periods for temperature (0-1, 2-7, 8-14, 15-21, and 22-28 days) and adjusted for calendar time (stratified by conurbation), relative humidity (average of lags $0-3)$, day of week, public holiday, influenza, respiratory syncytial virus, PM10 level (lags $0-3$ ), and ozone level (lags $0-3$ ). Reference value for relative risk estimates is mean value of daily mean temperature across all days included

The effects of day of week $(\mathrm{P}=0.13)$, holiday $(\mathrm{P}=0.16)$, influenza $(\mathrm{P}=0.96)$, respiratory syncytial virus $(\mathrm{P}=0.23), \mathrm{PM} 10$ levels $(\mathrm{P}=0.91)$, and ozone levels $(\mathrm{P}=0.56)$ did not vary across conurbations.

Effect modification by individual level factors

In a simplified model with a single temperature term (the average of lag days 0-28) evidence of effect modification by age was strong ( $\mathrm{P}$ for interaction $<0.001$ ); notably, adults aged 75-84 seemed more vulnerable to the effects of cold than other age groups including the eldest $(\geq 85)$ : relative risk per $1^{\circ} \mathrm{C}$ reduction in temperature was 1.016 (95\% confidence interval 1.007 to 1.025$)$ for those aged less than $65,1.018$ (1.009 to 1.027) for those aged 65-74, 1.027 (1.018 to 1.036) for those aged $75-84$, and 1.019 (1.009 to 1.029 ) for those aged 85 or more (fig 3). The temperature effect did not differ between men and women $(\mathrm{P}=0.80)$. Data were available on previous coronary heart disease (myocardial infarction or angina) for $82 \%$ of events, and those patients with previous disease seemed more vulnerable to the effects of temperature than those with no history of disease: relative risks per $1^{\circ} \mathrm{C}$ reduction in temperature 1.025 (1.015 to 1.034) and 1.019 (1.011 to $1.029)$; $\mathrm{P}$ for interaction 0.001 . However, there was little evidence of any effect modification by previous hypertension (P for interaction 0.16). Finally, considering the $86 \%$ of events when data on current use of aspirin were available, those taking aspirin seemed less vulnerable to the effects of temperature than those not taking aspirin: relative risk per $1^{\circ} \mathrm{C}$ reduction in temperature 1.016 (1.006 to 1.026$)$ compared with 1.022 (1.012 to 1.031); P for interaction 0.007. Effect modification by statin use was not assessed because of incomplete data.

\section{Sensitivity analyses}

To test the robustness of the effect estimates several modifications were made to the final model. The estimated overall temperature effect did not seem to be sensitive to restricting to only validated myocardial infarction events, using minimum and maximum daily temperature in place of mean temperature, restricting analyses to the five conurbations with the highest event rates, imputing pollution data to enable all observed data to be used, or including additional terms to allow for residual autocorrelation in the final model. For all of these sensitivity analyses the estimated cumulative effect of a $1^{\circ} \mathrm{C}$ reduction in temperature was between a $1.7 \%$ and $2.2 \%$ increase in risk of myocardial infarction, comparable to our final model estimate of a $2.0 \%$ increase in risk. Considering the temperature effect at specific lag periods, the effects of temperature at lag days 2-7 and 8-14 were estimated at $0.4-0.8 \%$ per $1^{\circ} \mathrm{C}$ reduction in temperature in all models, with no evidence of non-linearity in these effects. At shorter (0-1 day) and longer (15-21, 22-28 day) lag periods, the lack of evidence for a temperature effect was consistent across models.

Varying the level of seasonal control had only a small effect on the size and not direction of the estimated cumulative temperature effect: estimated effect 
Table 2 Estimated effects of temperature and potential confounders in final model

\begin{tabular}{|c|c|c|}
\hline Potential confounders & Relative risk $(95 \% \mathrm{Cl})$ & Pvalue \\
\hline \multicolumn{3}{|l|}{ Temperature (per ${ }^{\circ} \mathrm{C}$ drop): } \\
\hline Lag 0-1 & $1.002(0.998$ to 1.005$)$ & \multirow{5}{*}{$<0.001$} \\
\hline Lag 2-7 & $1.006(1.002$ to 1.011$)$ & \\
\hline Lag 8-14 & $1.007(1.003$ to 1.011$)$ & \\
\hline Lag 15-21 & $1.003(0.999$ to 1.007$)$ & \\
\hline Lag 22-28 & $1.002(0.998$ to 1.006$)$ & \\
\hline Cumulative effect over all lags & $1.020(1.011$ to 1.029$)$ & \\
\hline \multicolumn{3}{|l|}{ Relative humidity (\%): } \\
\hline 55 & $1.03(0.99$ to 1.06$)$ & \multirow{5}{*}{0.29} \\
\hline 65 & 1.01 (0.99 to 1.03$)$ & \\
\hline 75 & 1.00 (reference) & \\
\hline 85 & 1.01 (0.98 to 1.04$)$ & \\
\hline 95 & $1.06(0.99$ to 1.13$)$ & \\
\hline \multicolumn{3}{|l|}{ Day of week: } \\
\hline Sunday & 1.00 (reference) & \multirow{7}{*}{$<0.001$} \\
\hline Monday & 1.14 (1.11 to 1.17$)$ & \\
\hline Tuesday & 1.05 (1.02 to 1.08$)$ & \\
\hline Wednesday & 1.07 (1.04 to 1.10$)$ & \\
\hline Thursday & 1.04 (1.01 to 1.07$)$ & \\
\hline Friday & $1.08(1.05$ to 1.11$)$ & \\
\hline Saturday & 0.99 (0.96 to 1.02$)$ & \\
\hline \multicolumn{3}{|l|}{ Public holiday: } \\
\hline No & 1.00 (reference) & \multirow{2}{*}{0.21} \\
\hline Yes & 0.97 (0.92 to 1.02$)$ & \\
\hline \multicolumn{3}{|l|}{ Confirmed cases of influenza $A^{\star}$ : } \\
\hline 0 & 1.00 (reference) & \multirow{3}{*}{0.58} \\
\hline 1 & 1.02 (0.99 to 1.05$)$ & \\
\hline$\geq 2$ & $1.01(0.97$ to 1.05$)$ & \\
\hline
\end{tabular}

Confirmed cases of respiratory

syncytial virus*:

\begin{tabular}{|c|c|c|}
\hline 0 & 1.00 (reference) & \multirow{3}{*}{0.84} \\
\hline 1 & 0.99 (0.96 to 1.02$)$ & \\
\hline$\geq 2$ & 1.01 (0.95 to 1.06$)$ & \\
\hline \multicolumn{3}{|c|}{ PM10 $\left(\right.$ per $\left.\mu \mathrm{g} / \mathrm{m}^{3}\right) \dagger:$} \\
\hline Lag 0 & 1.001 (1.000 to 1.002$)$ & \multirow{4}{*}{0.02} \\
\hline Lag 1 & $0.999(0.998$ to 1.000$)$ & \\
\hline Lag 2 & $0.999(0.998$ to 1.000$)$ & \\
\hline Lag 3 & $1.000(0.999$ to 1.001$)$ & \\
\hline \multicolumn{3}{|c|}{ Ozone (per $\mu \mathrm{g} / \mathrm{m}^{3}$ ) } \\
\hline Lag 0 & $1.000(0.999$ to 1.001$)$ & \multirow{4}{*}{0.19} \\
\hline Lag 1 & $0.999(0.999$ to 1.000$)$ & \\
\hline Lag 2 & 1.000 (0.999 to 1.001$)$ & \\
\hline Lag 3 & 1.000 (0.999 to 1.000$)$ & \\
\hline
\end{tabular}

Model adjusted for season and trend using spline function of calendar date, with 7 knots per calendar year.

* Laboratory confirmed cases.

†Particulate matter with diameters less than $10 \mu \mathrm{m}$.

sizes were $1.2-1.4 \%$ for a $3-6$ degrees of freedom per year spline; $1.8-2.0 \%$ for $7-9$ degrees of freedom per year, and 1.0-1.4 for 10-14 degrees of freedom per year. Confidence intervals included our original estimate of a $2.0 \%$ increase in risk per $1^{\circ} \mathrm{C}$ reduction in temperature for all levels of seasonal control above 3 degrees of freedom per year.

\section{DISCUSSION}

Across the 15 conurbations in England and Wales included in our analyses we found a broadly linear association between daily mean ambient temperature and risk of myocardial infarction, with a $1^{\circ} \mathrm{C}$ reduction in temperature associated with a cumulative $2 \%(95 \%$ confidence interval $1.1 \%$ to $2.9 \%$ ) increase in risk of myocardial infarction over the current and subsequent 28 days. Because myocardial infarctions are common, and ambient temperature is experienced by the whole population, even a small increase in risk translates to substantial absolute numbers of extra myocardial infarctions. For example, in the United Kingdom, which has an estimated 146000 myocardial infarctions per year, ${ }^{19} 11600$ events would be expected on average in a 29 day period; our results suggest that each $1^{\circ} \mathrm{C}$ reduction in temperature nationwide on a single day would be associated with around 200 extra myocardial infarction events. We found no evidence of any detrimental heat effect.

\section{Comparison with other studies}

The effects of lower temperatures seemed to operate most strongly at two to 14 days after the reduction in temperature. The absence of a more immediate effect may be characteristic of the underlying mechanism at work, or might simply reflect delays in patients with myocardial infarction being admitted to hospital. A similarly delayed cold effect has also been seen for overall mortality ${ }^{16}$ although more immediate effects have also observed. ${ }^{1}$

In a recent systematic review of ambient temperature effects in studies with myocardial infarction outcomes, increases in risk of myocardial infarction at both higher and lower temperatures were reported by different studies, with four studies reporting both effects. ${ }^{1}$ However, a key finding of the review was the highly variable methodology among studies in this area to date; only a few controlled for air pollution and levels of infectious diseases such as influenza, or investigated lagged temperature effects beyond a few days; control for season and long term trend has also been inconsistent. Two studies that did address all of these methodological concerns were those of data on myocardial infarction related mortality, which found a detrimental effect of heat ${ }^{20}$ or of both heat and cold..$^{21}$ Such data may, however, have poor specificity because in a proportion of cases the myocardial infarction diagnosis is likely to be assumed; therefore these findings in reality may reflect temperature effects on more broad health outcomes. In contrast, of five studies that analysed validated myocardial infarction outcomes separately, only one based in a subarctic area reported a detrimental heat effect, ${ }^{22}$ most reporting an adverse effect only of cold. ${ }^{23-25}$ This is in keeping with the present analysis, which was based on an audit database in which $88 \%$ of myocardial infarction events were corroborated by an electrocardiograph trace, raised markers, or both, and in which our findings were robust to exclusion of the remaining $12 \%$ of events. 

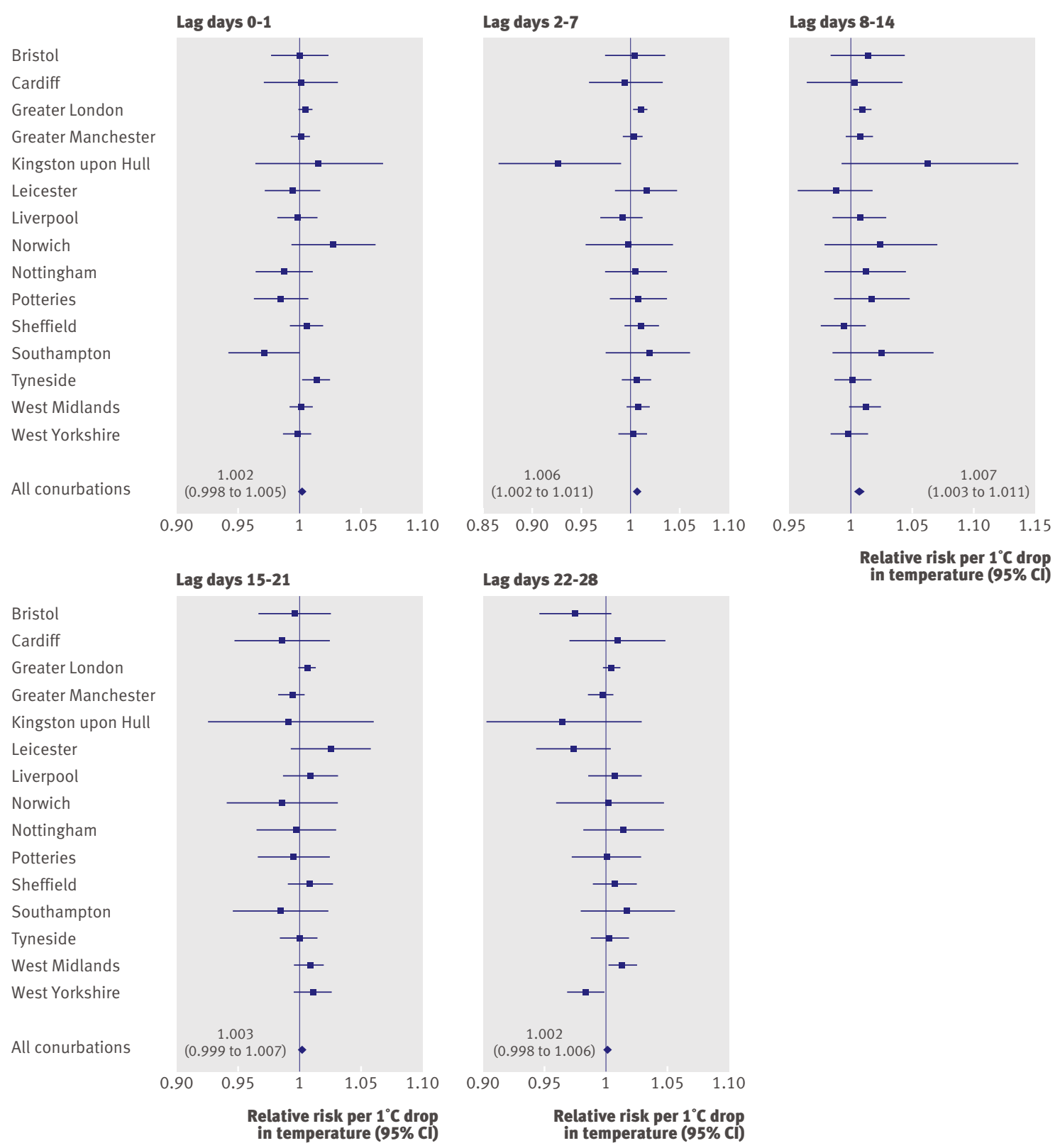

Fig 2 Estimated relative risk of myocardial infarction per $1^{\circ} \mathrm{C}$ reduction in temperature by conurbation. Estimates from a combined model including five temperature terms (lag days $0-1,2-7,8-14,15-21$, and 28), adjusted for calendar time (stratified by conurbation), and fixed effects across conurbations of relative humidity (average of lags 0-3), day of week, public holiday, influenza, respiratory syncytial virus, PM10 level (lags 0-3), and ozone level (lags 0-3)

We observed evidence of effect modification by age, with those aged 75-84 apparently more vulnerable to temperature effects than other age groups. Only a few studies have investigated the effects of temperature on risk of myocardial infarction by age group; one reporting an increased cold effect among those aged more than $65^{26}$ and a further two found no difference in effects when restricting their analyses only to older age groups. ${ }^{2324}$ The age structure of patients recorded in MINAP allowed us to subdivide those aged 65 or more into three age groups and it was of interest that an increased vulnerability did not extend into the oldest group ( 85 or more). A possible explanation is that people in this age group may spend less time outdoors and may be more likely to live in residential or nursing homes with effective heating systems. We also observed an increased vulnerability to cold among those with previous coronary heart disease, although we were not able to assess the three way interaction between temperature, age group, and previous coronary heart disease owing to low numbers of daily events in the combined subgroups; therefore whether the vulnerabilities owing to age and previous disease are independent remains an open question. People not taking aspirin also seemed to be more vulnerable to temperature effects. 


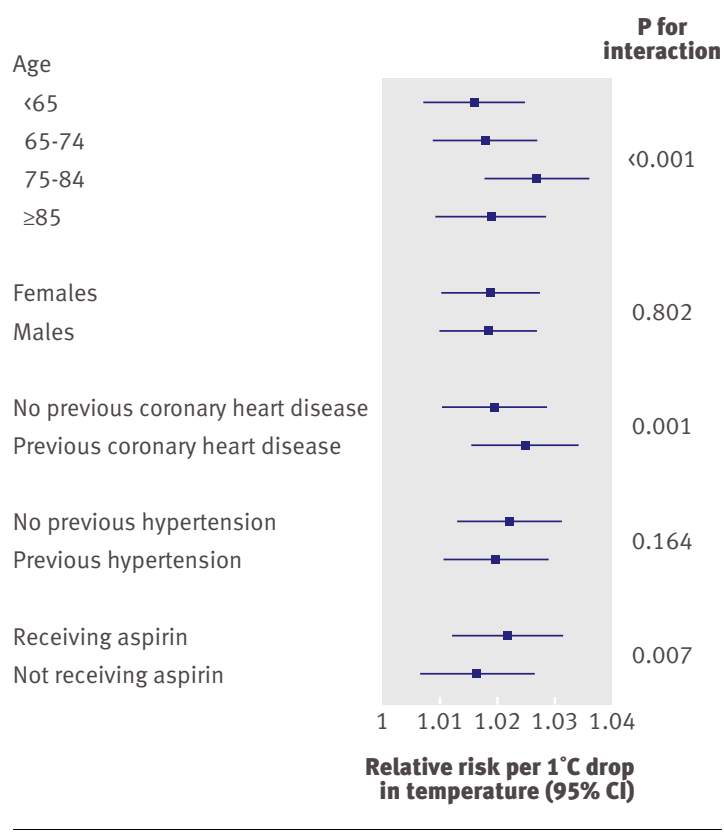

Fig 3 Estimated relative risk of myocardial infarction per $1^{\circ} \mathrm{C}$ reduction in temperature: effect modification by age, sex, history, and aspirin use. Graph shows estimated effect of temperature (average of lag days 0-28), adjusted for calendar time (stratified by conurbation), and fixed effects across conurbations of relative humidity (average lags 0-3), day of week, public holiday, influenza, respiratory syncytial virus, PM10 levels (lags 0-3), and ozone levels (lags 0-3)

\section{Policy implications and possible mechanisms}

Identifying subgroups that might be particularly vulnerable to cold effects is of interest since one potential application of our findings would be to inform a targeted early warning system based on forecasted weather, similar to that recently set up by the UK Meteorological Office for people with chronic obstructive pulmonary disease: when the risk of exacerbation of this disorder is increased because of low ambient temperature, people who are at risk are given advice through automated telephone calls, such as to keep warm, stay indoors, and watch for warning signs of their condition worsening; reductions in hospital admissions of between $15 \%$ and $76 \%$ have been reported among practices in the scheme. ${ }^{27}$ Our results suggest that for risk of myocardial infarction at colder temperatures, older people, those with coronary heart disease, and people who do not take aspirin could benefit from a similar targeted approach. However, since our findings imply an effect of temperature across the temperature range, and even among people outside the most vulnerable groups, a more widespread health education message aimed at reducing the impact of lower temperatures may be of value; such approaches should be evaluated.

Several mechanisms have been proposed to explain an effect of temperature reductions on risk of myocardial infarction. Exposure to cold under controlled conditions has been associated with increases in arterial pressure, blood viscosity, and cardiac workload. ${ }^{2829}$ A mobilisation of granulocytes has been observed, ${ }^{30}$ and red cell counts and plasma cholesterol and fibrinogen concentrations, all of which may be thrombogenic, seem to be raised after exposure to cold. ${ }^{2831}$ Finally, one study has suggested that the density distribution of blood platelet subpopulations may be affected, with an observed increase in less dense platelets that were more sensitive towards agents that induce aggregation. ${ }^{32}$ These small experimental studies combine to suggest that a pathway for cold induced thrombogenesis might involve a combination of factors, including haemoconcentration, an inflammatory response, and a tendency for an increased state of hypercoagulability. Furthermore, our observation that aspirin seemed to be partially protective suggests that part of the effect may be mediated by changes in platelet function. However, more recent data have been lacking and these hypothesised mechanisms need to be tested in larger studies examining a range of updated measures. Finally, it is possible that the observed effect of temperature reductions is not explained purely by direct biological pathways, but wholly or partly by other behaviour associated with lower temperatures - for example, increased snow shovelling activity might explain increases in risk of myocardial infarction at the lower extremes of temperature, ${ }^{33}$ although this would not explain the observed effect across the temperature range.

Interestingly, we did not detect any detrimental effect of temperature increases on risk of myocardial infarction. There are a few possible explanations for this. Firstly, temperature in the United Kingdom is rarely very high in global terms; although we included data from the unusually hot summer of 2003, even the warmest periods are brief, which may have limited our ability to detect a heat effect. Such heat effects, however, have been established in studies of overall mortality even in a UK setting. ${ }^{15} \mathrm{~A}$ second possibility is that any heat effects might have been too immediate to be detected by a daily time series study; data at a finer temporal resolution would be of interest. Finally, our results might simply reflect a genuine absence of any heat effect on myocardial infarction, indicating that other mechanisms are more important drivers of the heat-mortality relation; this is also indicated by mortality data from London that showed no increase in mortality from myocardial infarction at higher temperatures despite clear heat related increases in other cardiovascular deaths. ${ }^{34}$

\section{Strengths and limitations of the study}

Our study has some limitations. Firstly, the MINAP database is restricted to patients admitted to hospital; we therefore would not have included myocardial infarctions leading to death before hospital admission. The likelihood of a patient with myocardial infarction surviving to be admitted to hospital could conceivably be related to temperature if, for example, bad weather led to a delay in the ambulance. If such a mechanism were operating, however, it seems likely that the number of myocardial infarctions would then be 


\section{WHAT IS ALREADY KNOWN ON THIS TOPIC}

Ambient outdoor temperature has been shown to affect mortality risk in the short term, with both hot and cold days associated with increased mortality

The effect of ambient temperature on the risk of myocardial infarction is unclear

\section{WHAT THIS STUDY ADDS}

In urban settings in England and Wales, a lower daily mean temperature was associated with an increased risk of myocardial infarction; no increased risk was detected at higher temperatures

Each $1^{\circ} \mathrm{C}$ reduction in temperature in the United Kingdom on a single day would be associated with around 200 extra myocardial infarction events

Older people (185) and those with previous coronary heart disease seemed most vulnerable to the effects of temperature reductions; those taking prophylactic aspirin were less vulnerable likely to be the most vulnerable. Finally, studies of specific public health interventions aimed at reducing the impact of temperature related increases in risk of myocardial infarction are needed.

Contributors: $\mathrm{KB}, \mathrm{SH}, \mathrm{AH}, \mathrm{PW}$, and LS designed the study. $\mathrm{KB}$ and $\mathrm{EH}$ prepared and cleaned the data. KB did the statistical analysis and wrote the first draft. SH, AH, EH, PW and LS contributed to further drafts. KB and LS are the guarantors.

Funding: This study was funded through grants from the British Heart Foundation (FS/04/045) and the Garfield Weston Trust. LS is supported by a Wellcome Trust senior research fellowship in clinical science. SH is funded by a Wellcome Trust research career development fellowship (076583/Z/05/Z). The funders had no role in the design or conduct of this review, or in the preparation, review, or approval of the manuscript. All authors carried out this research independently of the funding bodies. Competing interests: All authors have completed the unified competing interest form at www.icmje.org/coi_disclosure.pdf (available on request from the corresponding author) and all authors want to declare: (1) financial support for the submitted work from the British Heart Foundation, the Garfield Weston Trust, and the Wellcome Trust; (2) no financial relationships with commercial entities that might have an interest in the submitted work; (3) no spouses, partners, or children with relationships with commercial entities that might have an interest in the submitted work; and (4) no non-financial interests that may be relevant to the submitted work.

Ethical approval: This study was approved by the London School of Hygiene and Tropical Medicine ethics committee.

Data sharing: No additional data available.

furthermore since our data were restricted to Englan and Wales we were unable to assess the hypothesis that temperature effects may vary with latitude and local climate. $^{7}$ Finally, data were not widely available on levels of particulates with a diameter less than $2.5 \mu \mathrm{m}$ (PM2.5), which may be a more important predictor of risk of myocardial infarction than PM10, ${ }^{14}$ therefore we cannot exclude residual confounding, although in the one monitoring station where both measures were recorded the correlation was high (0.92), so we believe that including PM10 in our models should have accounted for any major confounding effects of finer particulates.

Despite these limitations, to our knowledge this is the first large study to investigate the short term effects of ambient temperature on risk of myocardial infarction in which key potential confounders (air pollution, influenza activity, seasonality, and long term trend) were controlled for, non-linear effects were investigated, and in which most cases of myocardial infarction could be validated against electrocardiographic or marker criteria to confirm the diagnosis. In addition a range of sensitivity analyses confirmed that our main conclusions were robust to changes in aspects of the model specification, and to restricting to only the most "reliable" data.

\section{Conclusions}

In conclusion, our study shows a convincing short term increase in risk of myocardial infarction associated with lower ambient temperature, predominantly operating in the two weeks after exposure. International studies with consistent methods will be required to clarify the dependence of these effects on local climate, whereas individual level studies collecting demographic, clinical, and behavioural data may shed light on the role of adaptive measures such as clothing and home heating, and further clarify which subgroups are
1 Curriero FC, Heiner KS, Samet JM, Zeger SL, Strug L, Patz JA. Temperature and mortality in 11 cities of the eastern United States. Am J Epidemiol 2002;155:80-7.

2 Baccini M, Biggeri A, Accetta G, Kosatsky T, Katsouyanni K, Analitis A, et al. Heat effects on mortality in 15 European cities. Epidemiology 2008;19:711-9.

3 McMichael AJ, Wilkinson P, Kovats RS, Pattenden S, Hajat S, Armstrong B, et al. International study of temperature, heat and urban mortality: the 'ISOTHURM' project. Int J Epidemiol 2008;37:1121-31.

4 Analitis A, Katsouyanni K, Biggeri A, Baccini M, Forsberg B, Bisanti L, et al. Effects of cold weather on mortality: results from 15 European cities within the PHEWE project. Am J Epidemio 2008;168:1397-408.

5 Braga ALF, Zanobetti A, Schwartz J. The effect of weather on respiratory and cardiovascular deaths in 12 US cities. Environ Health Perspect 2002;110:859-63.

6 Donaldson GC, Keatinge WR. Early increases in ischaemic heart disease mortality dissociated from and later changes associated with respiratory mortality after cold weather in south east England. J Epidemiol Community Health 1997;51:643-8.

7 Bhaskaran K, Hajat S, Haines A, Herrett E, Wilkinson P, Smeeth L. Effects of ambient temperature on the incidence of myocardial infarction. Heart 2009;95:1760-9.

8 Doherty RM, Heal MR, Wilkinson P, Pattenden S, Vieno M, Armstrong B, et al. Current and future climate- and air pollutionmediated impacts on human health. Environmental Health 2009;8(suppl 1):8S

9 Ruckerl R, Greven S, Ljungman P, Aalto P, Antoniades C, Bellander T, et al. Air pollution and inflammation (interleukin-6, C-reactive protein, fibrinogen) in myocardial infarction survivors. Environ Health Perspect 2007;115:1072-80.

10 Dominici F. Time-series analysis of air pollution and mortality: a statistical review. Res Rep Health Eff Inst 2004:3-27.

11 McCullagh P, Nelder JA. Generalized linear models. 2nd ed. Chapman and Hall/CRC, 1989 pollution and infant mortality: analysis of daily time-series data in 10 English cities. J Epidemiol Community Health 2007;61:719-22.

13 Hajat S, Kovats RS, Lachowycz K. Heat-related and cold-related deaths in England and Wales: who is at risk? Occup Environ Med 2007;64:93-100.

14 Bhaskaran K, Hajat S, Haines A, Herrett E, Wilkinson P, Smeeth L. Effects of air pollution on the incidence of myocardial infarction. Heart 2009;95:1746-59.

15 Hajat S, Armstrong BG, Gouveia N, Wilkinson P. Mortality displacement of heat-related deaths: a comparison of Delhi, Sao Paulo, and London. Epidemiology 2005;16:613-20.

16 Pattenden S, Nikiforov B, Armstrong BG. Mortality and temperature in Sofia and London. J Epidemiol Community Health 2003;57:628-33.
12 Hajat S, Armstrong B, Wilkinson P, Busby A, Dolk H. Outdoor air 
17 Armstrong B. Models for the relationship between ambient temperature and daily mortality. Epidemiology 2006;17:624-31.

18 Brumback B, Ryan LM, Schwartz JD, Neas LM, Stark PC, Burge HA. Transitional regression models, with application to environmental time series. J Am Stat Assoc 2000;95:16-27.

19 Allender S, Peto V, Scarborough P, Rayner M. Coronary heart disease statistics. British Heart Foundation, 2008.

20 Rossi G, Vigotti MA, Zanobetti A, Repetto F, Gianelle V, Schwartz I. Air pollution and cause-specific mortality in Milan, Italy, 1980-1989. Arch Environ Health 1999;54:158-64.

21 Sharovsky R, Cesar LAM, Ramires JAF. Temperature, air pollution, and mortality from myocardial infarction in Sao Paulo, Brazil. Braz J Med Biol Res 2004;37:1651-7.

22 Messner T, Lundberg V, Wikstrom B. A temperature rise is associated with an increase in the number of acute myocardial infarctions in the subarctic area. Int J Circumpolar Health 2002;61:201-7.

23 Danet S, Richard F, Montaye M, Beauchant S, Lemaire B, Graux C, et al. Unhealthy effects of atmospheric temperature and pressure on the occurrence of myocardial infarction and coronary deaths. A 10year survey: the Lille-World Health Organization MONICA project (monitoring trends and determinants in cardiovascular disease). Circulation 1999;100:E1-7.

24 Enquselassie F, Dobson AJ, Alexander HM, Steele PL. Seasons, temperature and coronary disease. Int J Epidemiol 1993;22:632-6.

25 Wang H, Matsumura M, Kakehashi M, Eboshida A. Effects of atmospheric temperature and pressure on the occurrence of acute myocardial infarction in Hiroshima City, Japan. Hiroshima J Med Sci 2006;55:45-51.

26 Morabito M, Crisci A, Grifoni D, Orlandini S, Cecchi L, Bacci L, et al. Winter air-mass-based synoptic climatological approach and hospital admissions for myocardial infarction in Florence, Italy. Environ Res 2006;102:52-60.

27 UK Meteorological Office. COPD health forecasting winter 2005/6summary of benefits. www.metoffice.gov.uk/health/ evaluation_report_2005-06.pdf.

28 Keatinge WR, Coleshaw SR, Cotter F, Mattock M, Murphy M, Chelliah R. Increases in platelet and red cell counts, blood viscosity, and arterial pressure during mild surface cooling: factors in mortality from coronary and cerebral thrombosis in winter. $B M$ J 1984;289:1405-8.

29 Raven PB, Niki I, Dahms TE, Horvath SM. Compensatory cardiovascular responses during an environmental cold stress, 5 degrees C. J Appl Physiol 1970;29:417-21.

30 Mercer JB, Osterud B, Tveita T. The effect of short-term cold exposure on risk factors for cardiovascular disease. Thromb Res 1999;95:93-104.

31 Neild PJ, Syndercombe-Court D, Keatinge WR, Donaldson GC, Mattock M, Caunce M. Cold-induced increases in erythrocyte count, plasma cholesterol and plasma fibrinogen of elderly people without a comparable rise in protein C or factor X. Clin Sci (Lond) 1994;86:43-8

32 Opper C, Hennig J, Clement C, Laschefski U, Dey D, Dieckwisch J, et al. Lowering of body temperature affects human platelet functions and norepinephrine release. Pharmacol Biochem Behav 1995;51:217-21.

33 Heppell R, Hawley SK, Channer KS. Snow shoveller's infarction. BM 1991;302:469-70.

34 Fenn B, Hajat S, Wilkinson P. Mechanistic insights: cardiovascular events during hot weather. Epidemiology 2008;19:S172.

Accepted: 1 June 2010 